\title{
Efficacy of Oral Tolerance Induction to raw apple using an Ultra Rush protocol in 28 patients allergic to rosaceae fruits: RAAP Study
}

\author{
Michel Bouvier ${ }^{1}$, Marion Hacker², and Sébastien lefevre ${ }^{3}$ \\ ${ }^{1}$ Allergology Center of Beaujolais \\ ${ }^{2}$ Centre Hospitalier Lyon-Sud \\ ${ }^{3}$ Universite de Lorraine
}

September 11, 2020

\section{Efficacy of Oral Tolerance Induction to raw apple using an Ultra-Rush protocol in 28 patients allergic to rosaceae fruits: RAAP Study}

To the Editor,

The diagnosis of pollen-food allergy syndrome (PFAS) or oral allergy syndrome (OAS) relies on clinical history together with demonstration of allergen-specific IgE. PFAS is mainly observed in central and northern Europe. These symptoms follow the consumption of raw fruits ${ }^{1}$ and last 30 minutes, then stop ; but they tend to worsen over time, leading patients to avoid eating raw rosaceae fruit and many other raw fruits. This clinical presentation evolves concurrently with birch pollen allergy ${ }^{2}$. Over $70 \%$ of patients with birch allergy suffer from PFAS to rosaceae, particularly to raw apple ${ }^{3}$. Therapeutic management is limited ${ }^{4}$, based on avoidance of trigger foods ${ }^{5}$.

The results of betulaceae pollen immunotherapy remain limited ${ }^{6}$. Raw Golden apple (RGA) desensitization has already been described by NUCERA et al. in $2010^{7}$ in 2 patients. Likewise, RGA tolerance induction was described in 2012 by KOPAC et $\mathrm{al}^{8}$ and in 2014 by BOUVIER et $\mathrm{al}^{9}$ according to slow protocols where the maintenance dose was reached over several months.

We realized a retrospective observational impact study to present an accelerated protocol or ultra-rush (UR) for resumption of RGA intake performed in two private hospitals of the Lyon region (France), the Charcot clinic and the Beaujolais clinic, on 28 patients hospitalized between June 2016 until October 2017. The study was appropriately registered (ClinicalTrials.gov Identifier: NCT04116580).

Eligible patients were 10 years of age and older with allergic rhinitis related to PFAS for raw rosacea with avoidance of this family of fruits for at least 6 months. Subjects were excluded from the study in case of severe allergic reaction to rosacea or uncontrolled asthma with FEV1 $<70 \%$. The medical procedure consisted of 4 phases. During the first consultation, each patient's medical history was collected. Prick tests with airborne allergen and raw golden apple (RGA) were performed according to the prick-prick technic ${ }^{10}$; specific IgE for apple, rBet v 1 , rBet v $2 \backslash$ sout, rPru p 3 were prescribed. In total the inclusion of patients was based on their clinical history.

For the UR, patients were not on antihistamines. At time 0 (T0), prick test were performed with $\mathrm{RGA}^{9}$. At T0 +20 minutes, patients took $1 \mathrm{~g}$ of unpeeled RGA, then by doubling regularly the doses from $2 \mathrm{~g}$ to $128 \mathrm{~g}$, to reach a $255 \mathrm{~g}$ cumulative dose within in 3 hours. 
Then, half of an unpeeled RGA was consumed daily at home for one month, and one whole RGA daily the following month. For the patients who underwent the UR procedure between mid-April and mid-July, intake of other raw rosaceae was permitted at the end of week 5 post UR. A two-month evaluation consultation was conducted. Afterwards, patients had to maintain their tolerance by consuming half a raw apple of any type three times a week. A telephone interview was performed at 1 year of the UR to evidence tolerance and compliance.

FIGURE 1 Shematic overview of the Ultra-Rush protocol

TABLE 1 Population profile - Allergologic and Biological results

\begin{tabular}{|c|c|c|c|c|c|c|c|c|c|c|c|}
\hline $\mathrm{n}$ & $\mathrm{n}$ & Sex & Age & $R C$ & $A$ & $D A$ & $\begin{array}{l}I g E \\
\text { Bet } v 1 \\
k U A / l\end{array}$ & $\begin{array}{l}I g E \\
\text { Bet v2 } \\
k U A / l\end{array}$ & $\begin{array}{l}I g E \\
\operatorname{Pru} p \\
3 \\
k U A / l\end{array}$ & $\begin{array}{l}I g E \\
\text { Apple } \\
K U A / l\end{array}$ & $\begin{array}{l}\text { Prick } \\
\text { Apple } \\
(\mathrm{mm})\end{array}$ \\
\hline 1 & $W$ & $W$ & 24 & 1 & 1 & 1 & 60,5 & $<0,10$ & $<0,10$ & 7,16 & 10 \\
\hline 2 & $W$ & $W$ & 25 & 1 & 0 & 0 & 8,7 & 4,18 & $<0,10$ & 3,22 & 3 \\
\hline 3 & $W$ & $W$ & 39 & 1 & 0 & 0 & 70,5 & $<0,10$ & $<0,10$ & 1,04 & 7 \\
\hline 4 & $W$ & $W$ & 12 & 1 & 0 & 0 & $>100$ & $<0,10$ & $<0,10$ & 14,38 & 8 \\
\hline 5 & $W$ & $W$ & 25 & 1 & 0 & 0 & 12,6 & $<0,10$ & $<0,10$ & 1,37 & 6 \\
\hline 6 & $W$ & $W$ & 32 & 1 & 0 & 0 & 99,3 & $<0,10$ & $<0,10$ & 3,09 & 6 \\
\hline 7 & $W$ & $W$ & 23 & 1 & 0 & 0 & 19,6 & $<0,10$ & $<0,10$ & 1,18 & 8 \\
\hline 8 & $W$ & $W$ & 27 & 1 & 0 & 0 & 18,3 & $<0,10$ & $<0,10$ & 2,26 & 13 \\
\hline 9 & $M$ & $M$ & 39 & 1 & 0 & 0 & 70 & $<0,10$ & 4,43 & 4,43 & 8 \\
\hline 10 & $W$ & $W$ & 46 & 1 & 1 & 0 & 0,53 & $<0,10$ & $<0,10$ & 0,31 & 6 \\
\hline 11 & $W$ & $W$ & 14 & 1 & 1 & 1 & 96,5 & $<0,10$ & $<0,10$ & 9,12 & 4 \\
\hline 12 & $W$ & $W$ & 47 & 1 & 0 & 1 & 13,5 & $<0,10$ & $<0,10$ & 0,49 & $\begin{array}{l}4 \\
5\end{array}$ \\
\hline 13 & $W$ & $W$ & 29 & 1 & 1 & 0 & 74,6 & 17,1 & 2,82 & 25,4 & 6 \\
\hline 14 & $W$ & $W$ & 35 & 1 & 0 & 1 & 11,3 & $<0,10$ & $<0,10$ & $<0,10$ & 0 \\
\hline 15 & $M$ & $M$ & 45 & 1 & 0 & 0 & 13,7 & 2,7 & $<0,10$ & 1,19 & 7 \\
\hline 16 & $W$ & $W$ & 19 & 1 & 1 & 0 & 20,4 & 8,12 & $<0,10$ & 3,41 & 6 \\
\hline 17 & $M$ & $M$ & 28 & 1 & 1 & 1 & 24,6 & 20,9 & $<0,10$ & 6,52 & 0 \\
\hline 18 & $W$ & $W$ & 28 & 1 & 1 & 0 & 1,16 & 5,3 & $<0,10$ & 1,11 & 5 \\
\hline 19 & $W$ & $W$ & 45 & 1 & 0 & 0 & 46,5 & $<0,10$ & $<0,10$ & 4,86 & 15 \\
\hline 20 & $W$ & $W$ & 10 & 1 & 1 & 0 & 9,36 & 11,4 & 0,20 & 1,71 & 5 \\
\hline 21 & $W$ & $W$ & 42 & 1 & 1 & 0 & 8,52 & $<0,10$ & 0,19 & 1,14 & 8 \\
\hline 22 & $W$ & $W$ & 38 & 1 & 0 & 1 & 14,3 & $<0,10$ & $<0,10$ & 1,93 & 5 \\
\hline 23 & $W$ & $W$ & 25 & 1 & 0 & 0 & 4,53 & $<0,10$ & $<0,10$ & 2,60 & 7 \\
\hline 24 & $W$ & $W$ & 36 & 1 & 1 & 1 & 37,1 & 3,22 & $<0,10$ & 2,13 & 8 \\
\hline 25 & $M$ & $M$ & 27 & 1 & 1 & 0 & 26,8 & 7,15 & $<0,10$ & 3,15 & 7 \\
\hline 26 & $W$ & $W$ & 51 & 1 & 1 & 0 & $>100$ & $<010$ & $<0,10$ & 2,07 & 7 \\
\hline 27 & $W$ & $W$ & 30 & 1 & 1 & 0 & 21,6 & 2,82 & $<0,10$ & 2,72 & 8 \\
\hline 28 & $M$ & $M$ & 16 & 1 & 1 & 1 & 79,5 & 1,5 & $<0,10$ & 4,24 & 8 \\
\hline$\mu$ & $W+M$ & $W+M$ & 30,6 & 1 & 0,5 & 0,25 & 37,99 & 7,67 & 1,91 & 4,15 & 6,64 \\
\hline
\end{tabular}

n: Population; W : Women ; M: Men; RC: Rhinoconjunctivitus; A: Asthma ; AD : Atopic Dermatitis ; $\mu$ : Average;

28 patients were included ( 23 women and 5 men); average age 30.6 years (10 to 51 years). All had birch pollen- related allergic rhino conjunctivitis, 14 of them were asthmatic.PFAS duration averaged out at 6.8 years $(1 / 2-20$ years) and occurred 8.6 years on average after respiratory symptoms for 20 patients. For 8 
patients, food allergy occurred the same year as rhinitis. 26 patients had a positive apple prick test. rBet v $1 \mathrm{IgE}$ remained positive for all patients with an average rate of $37.99 \mathrm{kU} / \mathrm{L}(0,53 \mathrm{kU} / \mathrm{L}$ to $100 \mathrm{kU} / \mathrm{L})$; this rate is independent of the severity of the symptoms. rBet $v 2 \operatorname{IgE}$ remained positive for 11 patients with an average of 7,67 kU/L; apple IgE positive for 27 patients with an average of $4.15 \mathrm{kU} / \mathrm{L} ; 4$ patients had $\mathrm{rPru}$ p 3 positive $\operatorname{IgE}(0.19 \mathrm{kU} / \mathrm{L}$ to $4.43 \mathrm{kU} / \mathrm{L})$. The rate is low and it is not a contraindication for performing UR. All patients reached the cumulative dose. Tolerance was very good for 26 patients; Two did not tolerate the last dose; the first had an episode of isolated vomiting; the second had lower lip oedema. No severe anaphylactic reaction was observed.

During the post-hospitalization phase, all patients were able to consume half of a RGA a day for one month, and all of them consumed one raw apple a day during the $2 \mathrm{~d}$ month. Adverse effects of PFAS were mainly concentrated during the first two weeks (W); $25.5 \%$ at W1, and $15.3 \%$ at W2 to finally fall to $0.02 \%$ at W3 and after. No serious allergic reaction was recorded. Eleven out of 12 patients who had the rush between May and July reconsidered other raw rosaceae at two months. 4 asthmatic patients followed the protocol during the birch pollination period without severe incident, no particular exacerbations were observed.

At one year, 2 patients were lost to follow-up. 5 had stopped the protocol, 2 for compliance difficulties, 2 for the persistence of an uncomfortable PFAS, and 1 for gastroparesis attacks. At least 21 patients or $75 \%$ consumed all rosaceae at 1 year.

We demonstrated in a cohort of 28 patients that resumption of RGA eating in patients with PFAS related to raw rosaceae associated with persistent and severe allergic rhinitis to birch with Bet $\mathrm{v} 1$ biological profile is possible with an UR protocol to reach a cumulative dose of $255 \mathrm{~g}$ in 3 hours. This simple, fast and safe protocol, is easy to administer and allows acquiring tolerance that is quickly maintained by the regular consumption of apple. The tolerance is good. This protocol allows consumption of other raw rosacea at the end of the week 5 if it is achieved between mid-April and mid-July which could be the best period to carry out the UR. It is likewise feasible during the birch pollination phase without destabilizing underlying asthma. In all, the results of this study are encouraging. The effects are very positive particularly on quality of life. The disappearance of the PFAS was maintained over time if patients continued to consume raw rosaceae fruit regularly.

\section{CONFLICTS OF INTEREST}

Dr Bouvier report personal fees from Alk Abello, Chiesi, Stallergene Greer, Menarini and AstraZeneca unrelated to this work.

Dr Hacker report personal fees from Alk Abello, and Stallergene Greer unrelated to this work.

Dr Lefevre has no conflicts of interest.

\section{ACKNOWLEDGEMENTS}

We gratefully acknowledge the aid of Mrs Maya Perrou (Capio-Ramsay research coordinator) for her precious support throughout the conduct of this study, Professor Frederic Berard for his help during our first work on raw apple tolerance induction and our assistant Mrs Melissa Mcmahon. We also wish to thank the nursing teams in the 2 clinics, particularly Mrs Adeline Ballu allergology referent at the polyclinic of Beaujolais.

\section{ETHICS COMMITTEE APPROVAL AND CONSENT TO PARTICIPATE}

The Ethics Committee's approval was obtained by the Lyon University Hospital. Consent to participate was collected by sending an information letter to each patient or their family and non-objection to collection of their personal data. The study complied with national ethical and regulatory requirements (including data protection).

Michel Bouvier ID ID $^{1,2}$

Marion Hacker ID ${ }^{3}$ 
Sébastien Lefevre ID ${ }^{4}$

${ }^{1}$ Allergology Center of Beaujolais, 69400 Limas, France

${ }^{2}$ Polyclinic of Beaujolais, 69400 Arnas, France

${ }^{3}$ Department of Allergology, Lyon-Sud Hospital, 69190 Pierre Bénite, France

${ }^{4}$ Regional Institute for Allergic and Environmental diseases 57085 Metz, France

Address for Correspondence : Michel Bouvier, Allergology Center of Beaujolais, 63 Chemin de la Croix de Fer 69400 Limas France

Tel : +33608099155

Fax : +33478720840

Email :dr.michel.bouvier@orange.fr

Key Words: apple allergy; rosacea allergy; oral allergy syndrome; tolerance induction; ultra-rush

\section{REFERENCES}

Muluk NB, Cingy C et al. Oral allergy syndrom. Am. Jo. Rhinology Allergy . 2018; 32:27-30.

Breitender H, Ebner C. Molecular an Biochemical classification of plant derived food allergens. J. Allergy Clin Immunol . 2000; 106: 27-36.

Klinglmayr E, Hauser M, Wopfner $\mathrm{N}$ et al. Identification of B-cell epitopes of Bet $\mathrm{v} 1$ involved in cross reactivity with food allergens.Allergy. 2009; 64: 647-651.

Mari A, Bellmer - Weber BK, Vieths S. The oral allergy syndrome improved diagnostic and treatment methods - Allergy Clin Immunol2005; 5: 267-273.

Werfel T, Asero R, Ballmer-Weber B.K, Worm M, Hoffmann-Sommergruber K et al. Position paper of the EAACI: Food Allergy due to immunological cross-reactions with common inhalant allergens. Allergy. 2015; 70: 1079-1090.

Incorvia C, Ridolo E, Pastorello E et al. Allergen immunotherapy for birch apple syndrome, what do we know. Immunotherapy. 2017; 9 (15): 1271-1278.

Nucera E, Aruanno A et al. Apple desensitization in two patients with PR 10 proteins allergy. Allergy. 2010; 65 (8): 1060-1061.

Kopac P; Rudin M; Pichler W.J. Continuous apple consumption induces oral tolerance in birch-pollen associated apple allergy. Allergy.2012; 67: 280-285.

Bouvier M, Van der Brempt X, Nosbaum A; Cordier JM; Nicolas JF; Berard F. Induction of oral tolerance in allergy to rosaceae. Revue Française d'Allergologie . 2014; 54: 127-133.

Rudeschko O, Fahlbusch B, Vieth S et al. Investigation of the stability of apple allergen extracts. Allergy. 1995; 50: 575-579. 